\title{
Genes commonly involved in acid tolerance are not overexpressed in the plant microsymbiont Mesorhizobium loti MAFF303099 upon acidic shock
}

\author{
Marta Laranjo • Ana Alexandre • Solange Oliveira
}

Received: 7 January 2014 / Revised: 26 May 2014 / Accepted: 27 May 2014 / Published online: 17 June 2014

(C) Springer-Verlag Berlin Heidelberg 2014

\begin{abstract}
Rhizobia are legume bacterial symbionts that fix nitrogen in the root nodules of plants. The aim of the present study was to investigate the global transcriptional response of rhizobia upon an acidic shock. Changes in the transcriptome of cells of Mesorhizobium loti strain MAFF303099 upon an acidic shock at $\mathrm{pH} 3$ for $30 \mathrm{~min}$ were analysed. From a total of 7,231 protein-coding genes, 433 were found to be differentially expressed upon acidic shock, of which 322 were overexpressed. Although most of the overexpressed genes encode hypothetical proteins, the two most represented Cluster of Orthologous Group (COG) categories are 'defence mechanisms' and 'transcription'. Differentially expressed genes are dispersed throughout the chromosome, with the exception of the symbiosis island, where most genes remain unchanged. A significant number of transcriptional regulators and $\mathrm{ABC}$ transporter genes are overexpressed. No overexpression of genes typically associated to acid tolerance in rhizobia, such as act and exo genes, was detected. Overall, this study suggests a transcriptional response to acidic shock of M. loti distinct from other rhizobia. Additional studies are in course to explore the role of some of the highly overexpressed genes
\end{abstract}

Electronic supplementary material The online version of this article (doi:10.1007/s00253-014-5875-4) contains supplementary material, which is available to authorized users.

M. Laranjo $\cdot$ A. Alexandre $\cdot$ S. Oliveira

ICAAM-Instituto de Ciências Agrárias e Ambientais Mediterrânicas, Universidade de Évora, Pólo da Mitra, Ap. 94, 7002-554 Évora, Portugal

M. Laranjo · A. Alexandre

IIFA-Instituto de Investigação e Formação Avançada, Universidade de Évora, Ap. 94, 7002-554 Évora, Portugal

S. Oliveira $(\bowtie)$

Departamento de Biologia, Universidade de Évora, Pólo da Mitra, Ap. 94, 7002-554 Évora, Portugal

e-mail: ismo@uevora.pt and to further elucidate the molecular bases of acid stress response.

Keywords Rhizobia · Microarray $\cdot$ Transcriptome $\cdot$ Stress · $\mathrm{pH} \cdot$ Acid

\section{Introduction}

Rhizobia are soil bacteria that can live as saprophytes or form nitrogen-fixing symbiosis with legumes. Rapid adaptation of bacteria to diverse environmental changes is carried out by a series of global regulatory networks, which are actually stress response systems that control the simultaneous expression of a large number of genes and respond to changes of temperature, $\mathrm{pH}$, nutrients, salts and oxidation (Alexandre and Oliveira 2013; Ron 2006).

The productivity of leguminous crops is strongly affected by soil acidity, which may be natural or result from agricultural practices and industrial pollution. The problem with acidic soils is the limited availability of some essential plant nutrients (e.g. calcium and molybdenum) and the toxic levels of heavy metals, such as aluminium or manganese (Graham and Vance 2000). Soil acidity problems may be overcome by developing legume-rhizobia associations able to tolerate acidic soil conditions.

Environmental $\mathrm{pH}$ affects rhizobia survival and saprophytic growth in soil. Furthermore, rhizobia are usually more sensitive to low $\mathrm{pH}$ than legumes, which affects the establishment of symbiosis (Zahran 1999). Knowledge of pH-stress survival mechanisms is important for the development of rhizobia strains that will better survive soil acidity thus improving crop yields.

Mesorhizobium species nodulate a very diverse set of legume species, including the model legume, Lotus japonicus, and an important legume in human diet, Cicer arietinum 
(Laranjo et al. 2014). The optimal pH described for mesorhizobia is between 6 and 8 and the $\mathrm{pH}$ range is 4 to 10 (Chen et al. 2005). In general, low $\mathrm{pH}$ seems stressful for mesorhizobia; however, Mesorhizobium loti MAFF303099 is particularly tolerant to $\mathrm{pH} 5$ (Laranjo and Oliveira 2011). Studies on the biogeography of chickpea mesorhizobia suggest that, in this genus, acid tolerance may be related to the $\mathrm{pH}$ of the isolate origin soil (Brígido and Oliveira 2013).

A mixture of constitutive and inducible strategies can contribute to bacterial survival in an acidic environment, namely, the removal of protons, alkalisation of the external environment, changes in the composition of the cell envelope, production of general stress proteins and chaperones, expression of transcriptional regulators, and responses to changes in cell density (Cotter and Hill 2003; Kanjee and Houry 2013).

Cell envelopes are the first barriers that protect bacteria from the surrounding environment and the concomitant stresses. Thus, the first line of defence against adverse environmental $\mathrm{pH}$ should minimize cell envelope permeability, either to prevent ingress of protons or their loss (Hall et al. 1995).

ATP-binding cassette $(\mathrm{ABC})$ transporters have been shown to be involved in acid tolerance, e. g. in Streptococcus pneumoniae (Martín-Galiano et al. 2005), playing an important role in cytoplasmic $\mathrm{pH}$ regulation. This extremely diverse class of transporters couple the energy of ATP hydrolysis to the translocation of solutes (small molecules as well as ions) across biological membranes. The basic unit of an ABC transporter consists of four core domains: two transmembrane domains and two ATP-binding domains (Higgins 2001).

In general, chaperones and proteases have been implicated in the response of bacteria to environmental changes. However, the role of important chaperone systems, such as GroEL and DnaK, in acid stress does not seem to be as crucial as described for the heat-shock response in many bacteria. For example, in Escherichia coli, the chaperones so far implicated in acid response are Hsp31, HdeA and HdeB (Kanjee and Houry 2013).

Most $\mathrm{pH}$ stress protection systems include a mechanism for sustaining cytoplasmic $\mathrm{pH}$, and some of these systems offer cross-protection to other stresses. In E. coli, there are three main independent acid resistance (AR) systems: the oxidative or glucose-repressed, the glutamate-dependent and the argininedependent AR system (Audia et al. 2001; Foster 2004).

Some bacterial species show an increased survival rate after challenge at lethal acid $\mathrm{pH}$ if they are first subjected to a period of mild sub-lethal acidic conditions, a process known as adaptive acid tolerance or acid tolerance response (ATR), which has been described in E. coli (Goodson and Rowbury 1989). The stationary phase alternative sigma factor, $\sigma^{\mathrm{S}}$ (also known as RpoS or sigma 38), is required for ATR in Salmonella typhimurium (Lee et al. 1995). The RpoS alternative sigma factor is also regulated under acidic shock at the transcriptional level in E. coli (Audia et al. 2001).
In E. coli, other genes found to be involved in acid stress response include gad (glutamic acid decarboxylase) and omp (outer membrane protein) genes (Foster 2001), as well as genes encoding components of the electron transport chain (cyo, ndh, sdh and nuo genes) (Kanjee and Houry 2013).

Studies on rhizobia acid stress response have been conducted in Ensifer medicae (Tiwari et al. 2004), Ensifer meliloti (de Lucena et al. 2010; Hellweg et al. 2009), Rhizobium tropici (Graham et al. 1994) and M. loti (Correa and Barneix 1997; Correa et al. 1999). Overall, these studies suggest that act (for acid tolerance) and exo (exopolysaccharide I biosynthesis) genes are commonly involved in acid response.

In rhizobia, acid tolerance mechanisms seem to involve regulation of cytoplasmic $\mathrm{pH}$, proton exclusion and/or extrusion (Graham et al. 1994) and exopolysaccharide (EPS) production (Correa et al. 1999). The quantity of EPS produced by rhizobia isolates from Cicer, Phaseolus, Leucaena and Melilotus species is positively correlated with acid tolerance, and it is postulated that EPS could modify the rhizobia microenvironment and so decrease the stresses induced by an acid soil (Cunningham and Munns 1984). However, in M. loti strains, the EPS amount seems to have no correlation with acid tolerance, as tolerant strains showed lower ability to produce EPS under acidic conditions (Correa and Barneix 1997).

Acid response in rhizobia involves a range of genes that are essential for growth at low $\mathrm{pH}$, including some that are specific to acid stress response, the act genes, such as actA, actP, $a c t R$, and $a c t S$, and exoR (exopolysaccharide regulatory protein) gene. At least three regulatory systems exist in rhizobia: the two-component sensor-regulator system, actSR, is essential for induction of the adaptive ATR; another system involves the low $\mathrm{pH}$-induced transcriptional regulator gene, $p h r R$ ( $\mathrm{pH}$ regulated), which may control other low $\mathrm{pH}-$ regulated genes; the third circuit controls the expression of a $\mathrm{pH}-$ regulated structural gene, lpiA (low $\mathrm{pH}$ inducible) (Glenn et al. 1999). In addition, a number of orphan proteins of unknown function whose concentration changes at low $\mathrm{pH}$ was described in rhizobia (Dilworth et al. 2001).

E. medicae WSM419 has at least two systems responding to low $\mathrm{pH}$ : the $p h r R$ system and the system regulating lpiA, which is specific to low $\mathrm{pH}$ (Dilworth et al. 2001). In addition, the $a c t S-a c t R$ sensor-regulator system, where $a c t S$ is a sensor histidine kinase transmembrane protein and $a c t R$ a transcription regulator protein, is essential for acid tolerance and expressed constitutively in E. medicae, regardless of the medium pH (Tiwari et al. 1996). Nevertheless, there are other genes whose expression is modulated by low $\mathrm{pH}$, such as for example nodA and nodF in Rhizobium leguminosarum (Richardson et al. 1988).

Acid tolerance in $M$. loti strains seems to involve constitutive mechanisms, like outer membrane permeability, as well as adaptive responses to the medium $\mathrm{pH}$, such as the stage of 
bacterial growth and differential protein expression (Correa and Barneix 1997). However, little is known about the genes involved in the response to acidic stress.

Global transcriptional analysis can contribute to our knowledge on the mechanisms involved in rhizobia response to acidity. Only two microarray studies involving acid stress have been performed, both in E. meliloti 1021 (de Lucena et al. 2010; Hellweg et al. 2009), an acid-sensitive strain (Laranjo and Oliveira 2011). These studies indicate that the response of E. meliloti to low $\mathrm{pH}$ is characterised by the upregulation of exo genes, involved in exopolysaccharide I biosynthesis, and downregulation of flagellar and chemotaxis genes.

The aim of the present study was to analyse the global transcriptional response of $M$. loti MAFF303099 following an acidic shock. To our knowledge, this is the first study on the global transcriptional response to acidic stress in Mesorhizobium.

\section{Materials and methods}

Bacterial strains and growth conditions

The bacterial strain used in this study was $M$. loti strain MAFF303099. For gene expression profiling, three independent cultures were grown overnight at $28^{\circ} \mathrm{C}$ in $\mathrm{YMB}$ (Vincent 1970) medium to an optical density of $0.3(540 \mathrm{~nm})$. A $10-\mathrm{ml}$ volume of cells was used for each treatment: cells subjected to a pH of 3 for 30 min (acidic shock with a 1-M HCl solution) and cells exposed to no $\mathrm{pH}$ change (control).

RNA isolation and processing

After the acidic shock, cells were harvested immediately and total RNA was extracted using RNeasy Mini Kit (Qiagen, Venlo, Netherlands) with DNase (Roche Applied Science, Penzberg, Germany) treatment following the manufacturer's instructions. Once absence of residual DNA was confirmed, concentration and purity were determined using a Nanodrop ND-1000 UV-visible spectrophotometer (Thermo Scientific, Waltham, USA). RNA integrity was checked with an Agilent 2100 Bioanalyser using a RNA Nano assay (Agilent Technologies, Santa Clara, USA).

\section{Microarray experiments}

Microarray experiments were performed at BIOCANTGenomics Unit (Cantanhede, Portugal) as a service.

Each microarray experiment was conducted in three biological replicates. The messenger RNA (mRNA) microarrays (Mesorhizobium loti MAFF303099 $40 \mathrm{~K}$ ) were carried out as described by the manufacturer (MYcroarray, Ann Arbor,
USA). The array has 20,450 probes, of which 7,231 are unique, covering $99.3 \%$ of all genes. Slide images were acquired using the DNA Microarray B Scanner (Agilent Technologies, Santa Clara, USA) and scanned with an intensity of $100 \%$ PTM in the green channel.

\section{Microarray analysis}

Data were extracted using the QuantArray software (Packard BioScience, Meriden, USA). The arrays were analysed using the BRB data analysis tools for Excel (Simon et al. 2007) and normalized using the median. These data were then used to identify the genes with differential expression using the MeV4.0 software package (Saeed et al. 2006). A statistical Student's $t$ test with a $p$ value threshold of 0.01 was applied. The data were deposited in the NCBI Gene Expression Omnibus (GEO) under the record number GSE43527.

DNAPlotter (Carver et al. 2009) was used to generate circular DNA maps showing transcriptomics data.

Validation of microarray data by quantitative real-time RT-PCR

DNA microarray data were validated by quantitative real-time reverse transcription PCR (RT-PCR). For reverse transcription, $1 \mu \mathrm{g}$ of total RNA from M. loti MAFF303099 was used. Complementary DNA (cDNA) was synthesized using Maxima $^{\circledR}$ First Strand cDNA Synthesis Kit (Thermo Scientific, Waltham, USA) according to the manufacturer's instructions. Primers used to amplify selected $M$. loti MAFF303099 genes (Table 1) were designed using Primer Express 3.0 software (Applied Biosystems, Carlsbad, USA). RT-PCR amplification mixtures used $2.5 \mathrm{ng}$ of template cDNA, $2 \times$ SYBR Green PCR Master Mix and $0.3 \mathrm{mM}$ of reverse and forward primers for each gene in a total volume of $25 \mu \mathrm{l}$. Reactions were performed using a model 7500 thermocycler (Applied Biosystems, Carlsbad, USA). The expression ratio of the target genes was determined relative to reference genes hisC, rpoA and sigA, which showed no variation in the transcript abundance under the experimental conditions used here. Relative quantification of gene expression by real-time RT-PCR was determined by applying the $\Delta \Delta \mathrm{Ct}$ method (Pfaffl 2001).

\section{Gene re-annotation}

Since the full-genome annotation available for $M$. loti strain MAFF303099 was published in 2000 (Kaneko et al. 2000), most of the genes found to be overexpressed encode unknown or hypothetical proteins. We used Blast2GO (Götz et al. 2008) to annotate all differentially expressed genes encoding unknown or hypothetical proteins and STRING (http://string$\mathrm{db} . \mathrm{org}$ ) to assign Clusters of Orthologous Groups (COGs) (http://www.ncbi.nlm.nih.gov/COG/) to these newly 
Table 1 Primers list used for validation of microarray data by real-time RT-PCR

\begin{tabular}{|c|c|c|}
\hline Locus tag & Gene & Primer sequence $\left(5^{\prime}-3^{\prime}\right)$ \\
\hline \multirow[t]{2}{*}{ mlr5786 } & hisC & fwd: GGATAGCGTGGCGATGATG \\
\hline & & rev: TTGAGCACCCTGCAACGTT \\
\hline \multirow[t]{2}{*}{ mlr0325 } & rроA & fwd: CCTCTATTCGCCCGTCAAGA \\
\hline & & rev: CGTCATGGTCAGCTTGTCATAGTC \\
\hline \multirow[t]{2}{*}{ ml12386 } & $\operatorname{sig} A$ & fwd: GCCCTCTGCTCGACCTTTCC \\
\hline & & rev: AGCATCGCCATCGTGTCCTC \\
\hline \multirow[t]{2}{*}{ mll3842 } & $\operatorname{cit} Z$ & fwd: AAAAGCGCTCGACACCTATCTG \\
\hline & & rev: CGAAGGTCGAGGCGTTGA \\
\hline \multirow[t]{2}{*}{ mll6578 } & fixK & fwd: TCGTTGCCGTCGCATTCT \\
\hline & & rev: GTTGCGTTTCCGAGTCGAA \\
\hline \multirow[t]{2}{*}{ mll6630 } & $f i x N$ & fwd: GAGCCTTTCCGACAGCATATGT \\
\hline & & rev: ACTTGTGTTGCGCAGAAGAACA \\
\hline \multirow[t]{2}{*}{ mll1528 } & - & fwd: TCACCAGGATCGCCAATTG \\
\hline & & rev: AGCAGCCGGCGAATGTC \\
\hline \multirow[t]{2}{*}{ mlr2394 } & groEL & fwd:GTCGTAGAGGGCATGCAATTC \\
\hline & & rev: GACGCGCATCTTGTCCTGAT \\
\hline \multirow[t]{2}{*}{ mll3429 } & $c l p B$ & fwd:GGAGCTTGTCGGCCTTGA \\
\hline & & rev: AAGCCCGAGCTTCTGCTTCT \\
\hline
\end{tabular}

annotated genes (Szklarczyk et al. 2011). MicrobesOnline Operon Predictions (www.microbesonline.org/operons/) was used for operon prediction (Price et al. 2005).

\section{Results}

Global changes in gene expression induced by acidic shock

From a total of 7,231 protein-coding genes present in the M. loti MAFF303099 genome, 433 genes (6 \%) were found to be differentially expressed after the acidic shock with an $M$ value distribution between 3.9 and -6.1 (Fig. 1 and Supplementary Fig. S1). Furthermore, upregulation dominates over downregulation: 322 genes were found to be overexpressed whereas only 111 were underexpressed. Genes were considered as differentially expressed if $p \leq 0.01$, considering an average false discovery rate (FDR) of 0.11 . Low $M$ values were considered, as long as $p \leq 0.01$, since low level changes in gene transcription, commonly disregarded, may be an important part of cells response, as pointed out by Wren and Conway (2006).

From the 7,231 genes annotated in the M. loti MAFF303099 genome, 6,702 are located in the chromosome, 320 in pMLa and 209 in pMLb (Kaneko et al. 2000). Replicon distribution analysis of the 433 differentially expressed genes showed that $400(92 \%)$ are chromosomal, $13(3 \%)$ are in pMLa and 20 (5\%) are in pMLb. Most differentially expressed genes are upregulated in all three replicons: 295 genes in the chromosome, 9 in pMLa and 18 in pMLb (Table 2 and Fig. 2). From the 111 downregulated genes upon the acidic shock, 105 are in the chromosome, 4 in pMLa and 2 in pMLb (Table 2 and Fig. 2). It is noteworthy that $10 \%$ of the pMLb genes are differentially expressed (and $90 \%$ of these are overexpressed), while the percentage is smaller for the other replicons (chromosome, $6 \%$; pMLa, $4 \%$ ).

Differentially expressed genes are dispersed throughout the chromosome, with the exception of the symbiosis island. The expression level of genes located in the symbiosis island remains essentially unaltered (Fig. 2a), with only eight genes (approximately $1 \%$ ) being differentially expressed, namely, three overexpressed and five underexpressed, among a total of 580 genes (Kaneko et al. 2000).

It was possible to assign COGs to 355 of the 433 differentially expressed genes according to predicted gene functions (Table 3 and Supplementary Table S1). Genes were distributed by all 21 functional (COG) categories included in the genome. Figure 3 summarizes the percentage of differentially expressed genes in each COG category represented on the microarray. About $5 \%$ of the genes not assigned to a $\mathrm{COG}$ were differentially expressed upon the acidic shock.

The COG categories with the largest percentage of overexpressed genes are 'Defence Mechanisms' (V) (6 out of 67 genes) and 'Transcription' (K) (53 out of 594 genes) (Fig. 3).

The COG category with the largest number of genes with a significantly decreased expression is 'general function prediction only' (R) (14 genes) followed by 'signal transduction mechanisms' $(\mathrm{T})$, 'energy production and conversion' $(\mathrm{C})$ and 'carbohydrate transport and metabolism' (G) (10 genes in each category).

To confirm data obtained by microarray analysis, we examined the expression of six genes (citZ, fixK, fixN, mll1528, groEL and $c l p B$ ) by real-time RT-PCR (Table 4). Based on the results obtained by microarray analysis, the genes selected for real-time RT-PCR were chosen among those overexpressed, underexpressed and not differentially expressed. Results obtained by quantitative real-time PCR are globally in agreement with microarray data (Table 4).

\section{Transcriptional regulators/sigma factors}

From the 433 genes found to be differentially expressed upon the acidic shock, 48 genes are annotated as transcriptional regulators, of which 42 were overexpressed. These correspond to approximately $12 \%$ of the transcriptional regulators encoded in the MAFF303099 genome.

The overexpressed regulator genes with the highest fold induction ( $M$ values between 3.2 and 2.6) belong to different families of transcriptional regulators, namely, ml15152 and mlr7736 from the MarR family, mll3694 encoding a FixK 
Fig. 1 Microarrays analysis of M. loti MAFF303099 upon the acidic shock. $M$ value distribution for the differentially expressed genes $(p \leq 0.01)$. Overexpressed genes (322) have positive $M$ values, while underexpressed genes (111) have negative $M$ values

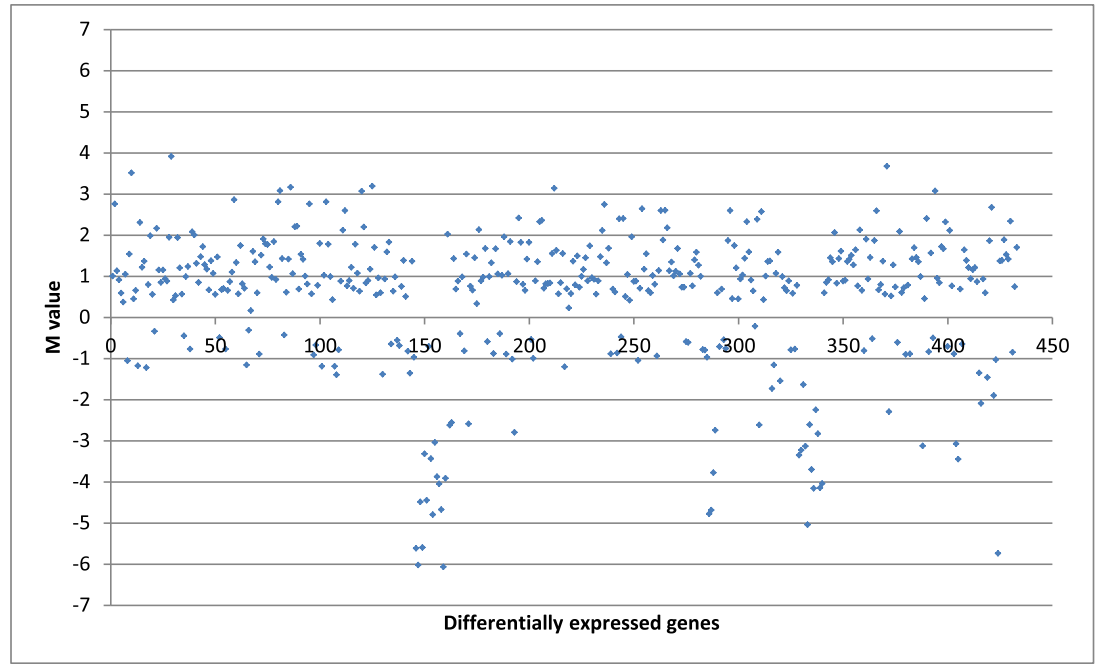

homologue, mll4997 from the LacI family, mll0250 from the IclR family and mll4816 from the TetR family.

Genes involved in cell envelope/ABC transporters

Gene mll1528 was found to be the most highly overexpressed gene $(M=3.9)$ upon the acidic shock. It encodes a small integral membrane protein with an iron permease conserved domain, which is probably part of an $\mathrm{ABC}$ transporter system. Several genes with an $M$ value higher than 2 upon the acidic shock (Table 3) are involved in 'cell wall/membrane/envelope biogenesis' and 'carbohydrate transport and metabolism'. For example, gene mll0693 $(M=3.5)$ encodes a sugar transferase involved in the cell envelope (outer membrane) biogenesis.

We have found at least 24 differentially expressed ABC transporter genes, of which 18 are upregulated ( $M$ values between 3.9 and 0.2 ). These 18 upregulated genes, likely to belong to 14 different $\mathrm{ABC}$ transporter systems, include five permeases and five ATP-binding proteins. Gene mll3590 ( $M=$ 3.1) encodes a periplasmic component of an ABC-type sugar transport system similar to the E. coli $u g p B$ gene. It is in the same operon as gene ml13591, which is also overexpressed $(M=1.4)$. Gene mll4997 $(M=3.1)$ encodes a periplasmic component of an ABC-type sugar transport system similar to the $E$. coli $r b s B$ gene. It is located upstream of the operon containing genes

Table 2 Number of genes overexpressed and underexpressed upon acidic shock, in each replicon

\begin{tabular}{lll}
\hline & $\begin{array}{l}\text { Number of } \\
\text { overexpressed genes }\end{array}$ & $\begin{array}{l}\text { Number of } \\
\text { underexpressed genes }\end{array}$ \\
\hline Chromosome & 295 & 105 \\
pMLa & 9 & 4 \\
pMLb & 18 & 2 \\
\hline
\end{tabular}

mll4996, mll4993 and mll4992, encoding an ABC-type sugar transport system. Gene mlr3639 $(M=2.6)$ encodes a trehalose-/maltose-binding protein, which is a periplasmic component of an ABC-type sugar transport system similar to the E. coli $u g p B$ gene and is part of an operon containing genes mlr3640, mlr3641, mlr3643, mlr3644 and mlr3645. The different induction levels found among the genes of some predicted operons may be due to internal regulation (promoters or terminators), premature termination or differential mRNA degradation (Laing et al. 2006).

Genes involved in exopolysaccharide biosynthesis

M. loti MAFF303099 genome has 32 genes annotated as involved in EPS synthesis. Among these, only one was found to be overexpressed, namely, exs $G$ (mlr3704), a sensory transduction histidine kinase $(M=1.9)$.

\section{Acid tolerance $(a c t)$ genes}

A cation transporting P-type ATPase (acid-induced copper pump) (mlr5325) with homology to actP is reported as overexpressed $(M=2.4)$. Other important act genes, such as the genes mlr5307 and mlr5308 coding for the twocomponent regulatory system ActS/ActR, respectively, or the apolipoprotein n-acetyltransferase gene actA (mlr5543), were found to be not differentially expressed.

Similarly, the transcription levels of the genes lpiA (mll8344) and phrR (mlr5544) remained unchanged.

Nitrogen fixation genes

fix genes are involved in nitrogen fixation and can code electron transport chains to nitrogenase, cytochrome oxidase and transcriptional regulators (Terpolilli et al. 2012). Nine fix 


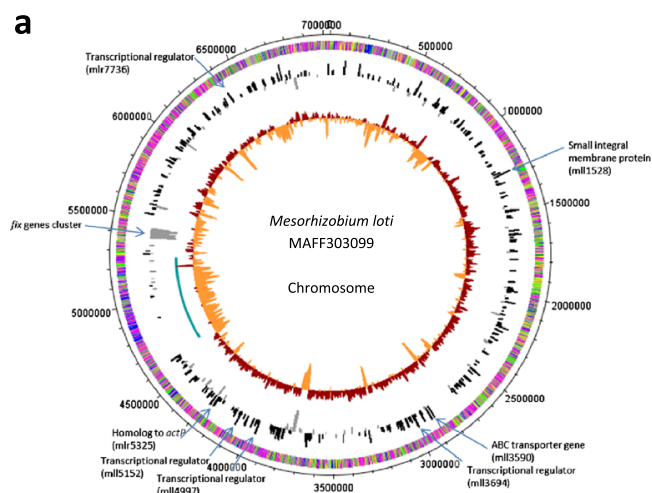

b

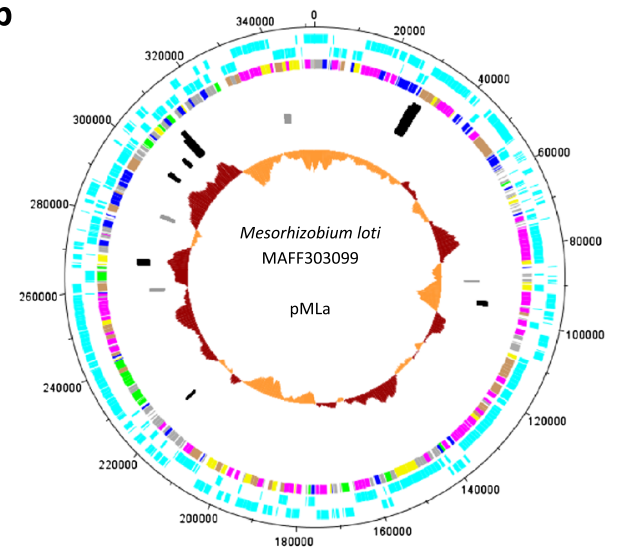

C

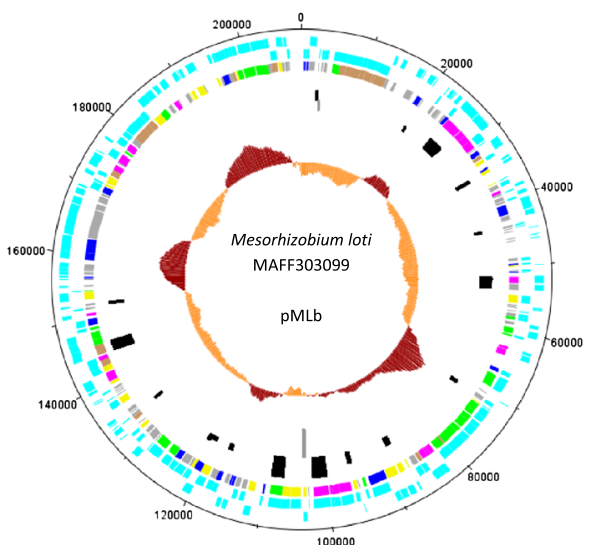

Fig. 2 Circular plots of the chromosome (a) and the two plasmids (b, c) from M. loti MAFF303099: outer ring, COG group for each gene; middle ring, acid shock transcriptome data ( $M$ values); inner ring, \%GC. Plasmid plots include two additional outer rings displaying genes encoded in the plus strand (outermost ring) and minus strand. COG colours: information storage and processing, blue; cellular processes and signalling, green; metabolism, magenta; poorly characterized, yellow; more than one COG category, brown; no COG, light grey. Transcriptome data: overexpressed (black) and underexpressed genes (grey). \%GC data: above average (red) and below average (orange). The symbiosis island is marked in blue in the chromosome plot (coordinates 4644792 to 5255766) (Kaneko et al. 2000). Some of the most relevant genes in the acid shock response are mapped in the chromosome plot (Color figure online) genes are found to be severely underexpressed: fixN (mll6630), fixK (mll6578), fixI (mll6624), fixO (mll6629), fixP (mll6628), fixG (mll6626), fixQ (msl6627), fixS (msl6623) and fixH (mll6625). All these genes are located in a chromosomal cluster outside the symbiosis island (coordinates 4644792 to 5255766 ) (Fig. 2a). However, some of these genes have multiple copies, such as fixH and fixNOPQ, which have one homologue within the symbiosis island, whose expression remained unaltered.

On the other hand, a second fixK homologue, mll3694, located in the chromosome but outside the symbiosis island, is upregulated $(M=3.2)$. FixK is a transcriptional regulator required for low $\mathrm{pH}$ induction of fix genes, which is regulated through ActR (Fenner et al. 2004).

Proteases and chaperones genes

No significant upregulation of proteases and chaperones genes was observed in the present microarray analysis, except for one serine protease gene (mlr7692) with homology to $\operatorname{deg} P 1$ that was found to be overexpressed $(M=1.8)$.

However, the expression values obtained with real-time PCR for groEL indicates its overexpression $(M=1.6)$, while for $\operatorname{clp} B$, a slight underexpression $(M=-0.5)$ was detected upon acidic shock.

\section{Discussion}

When subjected to the acidic shock, $M$. loti strain MAFF303099 showed 322 genes overexpressed and 111 underexpressed. All three replicons show a higher number of induced over repressed genes.

Similarly to the observed response of MAFF303099 following the acidic shock, also in E. meliloti strain 1021 (de Lucena et al. 2010; Hellweg et al. 2009), upregulation dominates over downregulation. The MAFF303099 transcriptional response included the overexpression of a significant number of genes encoding transcriptional regulators and $\mathrm{ABC}$ transporters.

Contrary to the response of E. meliloti to low $\mathrm{pH}$, in which a large number of exopolysaccharide biosynthesis genes are upregulated and motility and chemotaxis genes are downregulated (Hellweg et al. 2009), in strain MAFF303099, these genes were found to remain mostly unchanged. Only 1 out of 32 genes involved in EPS biosynthesis was found to be overexpressed. Our results suggest that the response of $M$. loti MAFF303099 to acidic stress does not include an increase in the synthesis of EPS. This hypothesis is supported by previous studies reporting that $M$. loti acid-tolerant strains showed a decreased production of EPS in acidic medium (Correa and Barneix 1997). Other authors had already reported a lack of 
Table 3 List of genes with higher increased expression $(M \geq 2)$ upon the acidic shock

\begin{tabular}{|c|c|c|c|c|}
\hline Locus tag & Location & Gene description & COG category $^{a}$ & M value \\
\hline mll1528 & Chr & Small integral membrane protein & $\mathrm{S}$ & 3.9 \\
\hline $\mathrm{mlr} 8209$ & $\mathrm{Chr}$ & Transcriptional family with acetyltransferase activity & $E / K$ & 3.7 \\
\hline mll0693 & $\mathrm{Chr}$ & Sugar transferase & M & 3.5 \\
\hline mll5152 & Chr & Transcriptional regulator & $\mathrm{K}$ & 3.2 \\
\hline mll3694 & $\mathrm{Chr}$ & Transcriptional regulator & $\mathrm{T}$ & 3.2 \\
\hline mlr0132 & $\mathrm{Chr}$ & Type 12 methyltransferase & $\mathrm{H}$ & 3.1 \\
\hline mll3590 & $\mathrm{Chr}$ & Sugar transporter sugar binding protein & G & 3.1 \\
\hline msl1808 & Chr & Hypothetical protein & - & 3.1 \\
\hline mll4997 & Chr & Family transcriptional regulator & G & 3.1 \\
\hline $\mathrm{mll} 2749$ & Chr & DMT superfamily & $\mathrm{R}$ & 2.9 \\
\hline mll3568 & Chr & Esterase lipase-like protein & $\mathrm{I}$ & 2.8 \\
\hline $\mathrm{mll} 4607$ & Chr & Ku protein & $\mathrm{S}$ & 2.8 \\
\hline mll0250 & Chr & Transcription regulator & $\mathrm{K}$ & 2.8 \\
\hline mll4093 & $\mathrm{Chr}$ & Transglutaminase domain protein & $\mathrm{E}$ & 2.8 \\
\hline mlr1995 & $\mathrm{Chr}$ & Hypothetical protein & not in COG & 2.7 \\
\hline msr4805 & Chr & Hypothetical protein & - & 2.7 \\
\hline $\mathrm{mlr} 3136$ & Chr & Hypothetical protein & not in COG & 2.6 \\
\hline $\mathrm{mlr} 3707$ & $\mathrm{Chr}$ & Hypothetical protein & not in COG & 2.6 \\
\hline $\mathrm{mlr} 3639$ & $\mathrm{Chr}$ & Trehalose/maltose binding protein & G & 2.6 \\
\hline mll4816 & Chr & Tetr family transcriptional regulator & $\mathrm{K}$ & 2.6 \\
\hline mlr4831 & $\mathrm{Chr}$ & Phenylalanine 4-monooxygenase ( $p p h a$ ) & E & 2.6 \\
\hline mlr7736 & Chr & Transcriptional regulator & K & 2.6 \\
\hline $\mathrm{mlr} 5354$ & Chr & Haloalkane dehalogenase & $\mathrm{R}$ & 2.6 \\
\hline mll8569 & Chr & Transcriptional regulator & $\mathrm{G} / \mathrm{K}$ & 2.4 \\
\hline mlr9679 & pMLb & Circadian oscillation regulator kaic-like protein & $\mathrm{L} / \mathrm{T}$ & 2.4 \\
\hline $\mathrm{m} \mid \mathrm{r} 2526$ & Chr & Methylated-DNA-protein-cystein methyltransferase & $\mathrm{L}$ & 2.4 \\
\hline $\mathrm{mlr} 2436$ & $\mathrm{Chr}$ & NADH-dependent dyhydrogenase & $\mathrm{R}$ & 2.4 \\
\hline $\mathrm{mlr} 5325$ & Chr & Cation transporting P-type atpase & $\mathrm{P} / \mathrm{S}$ & 2.4 \\
\hline mll9638 & pMLb & Serine protein & $\mathrm{T}$ & 2.4 \\
\hline msr8675 & $\mathrm{Chr}$ & Hypothetical protein & $\mathrm{s}$ & 2.3 \\
\hline mlr5096 & Chr & Phosphoenolpyruvate carboxykinase & $\mathrm{C}$ & 2.3 \\
\hline mll9633 & pMLb & Formate dehydrogenase & $\mathrm{C}$ & 2.3 \\
\hline $\mathrm{msl} 3831$ & Chr & Conserved hypothetical transmembrane protein & not in COG & 2.3 \\
\hline mll0857 & Chr & Transcriptional regulator & $\mathrm{K}$ & 2.3 \\
\hline mll3842 & $\mathrm{Chr}$ & Citrate synthase 2 & C & 2.2 \\
\hline mll3776 & Chr & Streptothricin-acteyl-transferase & $\mathrm{R}$ & 2.2 \\
\hline mll5048 & $\mathrm{Chr}$ & Family protein & $\mathrm{S}$ & 2.2 \\
\hline $\mathrm{mlr} 3777$ & Chr & Family transcriptional regulator & K & 2.2 \\
\hline mll1046 & Chr & Cytochrome C oxidase subunit I & $\mathrm{S}$ & 2.2 \\
\hline mll7529 & Chr & Glyoxalase bleomycin resistance protein dioxygenase & $\mathrm{R}$ & 2.1 \\
\hline mlr7359 & Chr & Transcriptional regulator & K & 2.1 \\
\hline mll4785 & Chr & NADPH:ferredoxin reductase & C & 2.1 \\
\hline msl5548 & Chr & Hypothetical protein & - & 2.1 \\
\hline mlr1797 & Chr & Conserved domain protein & $\mathrm{S}$ & 2.1 \\
\hline $\mathrm{m} \mid \mathrm{r} 9030$ & pMLa & Hydantoinase & $\mathrm{E} / \mathrm{Q}$ & 2.1 \\
\hline mll1959 & $\mathrm{Chr}$ & BA14K family protein & not in COG & 2.1 \\
\hline mlr7106 & Chr & Dehydrogenase & $\mathrm{I} / \mathrm{Q} / \mathrm{R}$ & 2.1 \\
\hline mll6735 & Chr & Arginine/ornithine antiporter & $\mathrm{E}$ & 2.0 \\
\hline mll1989 & Chr & Family transcriptional regulator & $\mathrm{K} / \mathrm{T}$ & 2.0 \\
\hline
\end{tabular}




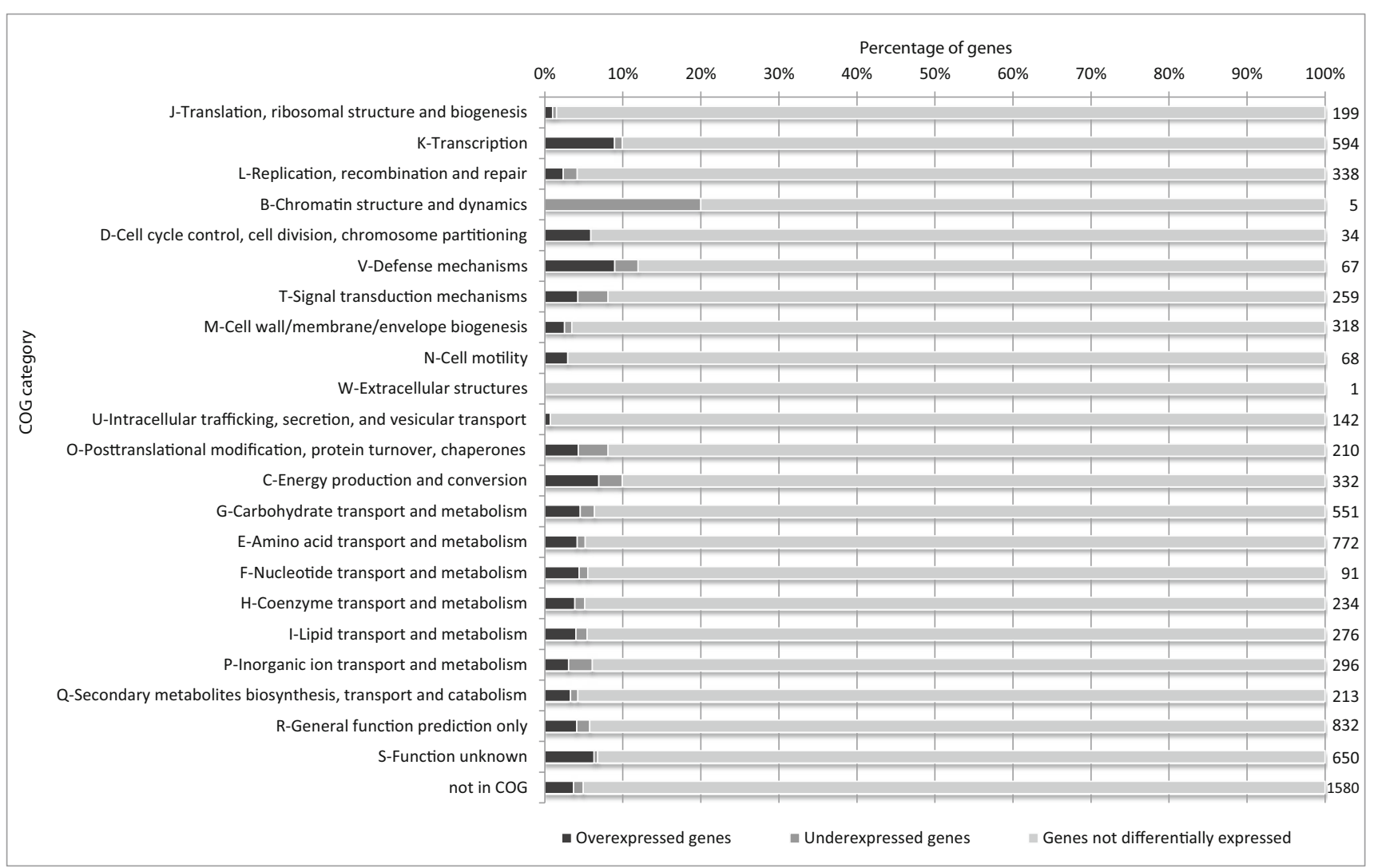

Fig. 3 Percentage of differentially expressed genes by COG category

correlation between exopolysaccharide synthesis and acid tolerance (Chen et al. 1993).

The different tolerance levels to low $\mathrm{pH}$ of $M$. loti MAFF303099 (tolerant) and E. meliloti 1021 (sensitive) results not only from their different gene content and regulation but also from their distinct transcriptional response to acidity. Since M. loti is acid-tolerant and shows no significant upregulation of genes previously described to be involved in acid tolerance, we may hypothesise a constitutive expression of these genes and/or an alternative mechanism of acid tolerance. Furthermore, comparisons should be carefully considered as

Table 4 Quantitative real-time RT-PCR analysis for validation of microarray data

\begin{tabular}{llll}
\hline Locus tag & Gene & \multicolumn{1}{l}{$M$ value } \\
\cline { 3 - 4 } & & Microarrays & Real-time PCR \\
\hline ml13842 & citZ & 2.2 & 3.4 \\
ml16578 & fixK & -6.0 & -6.0 \\
mll6630 & fixN & -6.1 & -6.7 \\
ml11528 & - & 3.9 & 1.4 \\
mlr2394 & groEL & n.d.e. & 1.6 \\
ml13429 & clpB & n.d.e. & -0.5 \\
\hline
\end{tabular}

n.d.e. not differentially expressed different stresses were applied in each study: E. meliloti 1021 was subjected to a $\mathrm{pH}$ shift from 7 to 5.75 for $60 \mathrm{~min}$ (Hellweg et al. 2009), while $M$. loti MAFF303099 suffered an acidic shock from $\mathrm{pH} 7$ to $\mathrm{pH} 3$, for $30 \mathrm{~min}$. However, Hellweg et al. (2009) have shown that genes are rapidly induced (or repressed) after an acidic shock, which suggests that the shock period per se cannot explain the different results obtained with these two rhizobia strains.

In our study, the transcriptional levels of most act genes, commonly involved in acid response in rhizobia, remained unaltered. Interestingly, in E. medicae WSM419, which is also an acid-tolerant strain, act $R$ and act $S$ are constitutively expressed irrespectively of the external $\mathrm{pH}$ (Tiwari et al. 1996).

Of the three known acidity sensor systems in root nodule bacteria (Dilworth et al. 2001), ActS-ActR, PhrR and LpiA, none of the encoding genes was found to be differentially expressed in our study, which suggests that the acid tolerance mechanisms in Mesorhizobium are probably different and rely on other genes.

In the present study, the unaltered expression of genes found to be involved in acid response in other rhizobia together with the high tolerance of MAFF303099 to acidity may suggest that these genes are constitutively expressed in $M$. loti MAFF303099 and/or other resistance mechanisms are involved. These might essentially comprise genes coding for 
transcriptional regulators and $\mathrm{ABC}$ transporter systems, since many of these genes were found to be upregulated in the present study.

All bacterial genomes encode an essential housekeeping sigma factor and most have at least one alternative sigma factor. Housekeeping sigma factors recognize a large set of promoters, while alternative sigma factors recognize specific groups of promoters for genes with a shared function (Gruber and Gross 2003). Since alternative sigma factors compete with the housekeeping sigma factor, changes in gene expression can occur by controlling expression, activity and availability of alternative sigma factors (Österberg et al. 2011). The presence of a large number of alternative sigma factors in a species appears to correlate with a diverse lifestyle (Gruber and Gross 2003). M. loti MAFF303099 has 25 putatively annotated sigma factors. Among these, at least eight are alternative sigma factors: three rpoN (nitrogen limitation), three rpoE (extracytoplasmic-ECF type) and two rpoH (heat shock) sigma factors. $\mathrm{RpoH} 2$ is an alternative sigma factor involved in the regulation of EPS synthesis in rhizobia, acting as a transcriptional regulator of the exo genes (Kaufusi et al. 2004). In M. loti MAFF303099, the $r p o H 2$ gene (mlr3862) was not found to be differentially expressed upon the acidic shock, which could probably explain the unaltered transcription level of almost all genes involved in EPS biosynthesis.

Bacterial survival in a medium with high proton concentration is dependent upon its ability to maintain a constant cytoplasmic $\mathrm{pH}$. This can partly be achieved by avoiding the diffusion of protons from the medium into the cell, so changes in envelope composition (phospholipid, fatty acid, and protein composition) may be an adaptation to survive at low $\mathrm{pH}$, as suggested for M. loti (Correa et al. 1999). In the present study, several genes coding for outer membrane and other cell envelope proteins, namely, $\mathrm{ABC}$ transporters, were reported as overexpressed, thus probably contributing to maintenance of the cell internal $\mathrm{pH}$.

Our results have shown a number of genes encoding transcriptional regulators of the MarR and TetR families to be overexpressed upon the acidic shock. These are repressors that control expression of genes coding for efflux pumps (Grkovic et al. 2002). marR regulator genes have been found to be induced by acidic stress in other bacteria such as Dickeya dadantii (Reverchon et al. 2010). Regulators from the MarR and TetR families have also been reported to be associated with oxidative stress response in Bradyrhizobium japonicum (Masloboeva et al. 2012).

Although some chaperones and proteases have been described to be involved in the acid response in bacteria, no significant upregulation of these genes was observed in the present microarray analysis. However, a previous study with several mesorhizobia species indicated that genes encoding important chaperones, such as DnaK and GroESL, are induced upon acidic shock in most acid-tolerant strains
(Brígido and Oliveira 2013). In the present study, the realtime RT-PCR analysis shows an induction of the groEL gene (mlr2394), although the microarray data indicated no differential expression. We cannot exclude the possibility that the inherent variability of microarray data may lead to some biologically important changes in gene expression being statistically excluded from the analysis, as described before in other bacteria (Martín-Galiano et al. 2005). Another chaperone, known to interact with the DnaKJ system, is ClpB. This chaperone gene was found to be not differentially expressed in the present study, and this is consistent with previous results in Mesorhizobium ciceri that showed that the $\operatorname{clp} B$ knockout did not affect the ability of the mutant strain to tolerate an acidic shock (Brígido et al. 2012). Agreeing with previous studies that had shown the protease gene $\operatorname{deg} P$ as the most strongly induced gene in E. meliloti upon acidic shift (de Lucena et al. 2010), our results have shown overexpression of a serine protease gene with homology to $\operatorname{deg} P$, a secondary response mechanism, which breaks down misfolded proteins that could not be recovered by the chaperones, when the external $\mathrm{pH}$ is very low.

A recent study reported the transcriptome analysis of $M$. loti MAFF303099 when subjected to heat shock (Alexandre et al. 2014). A global comparison of these two responses to stress shows that the number of genes differentially expressed is much smaller in the case of acidic shock, and moreover, the response to acid conditions is mostly driven by gene upregulation, while the heat shock led to an extensive gene downregulation.

Comparing this study in M. loti MAFF303099 with two previous studies in E. meliloti 1021 (de Lucena et al. 2010; Hellweg et al. 2009), distinct genes were found to be overexpressed. Our results suggest that the response mechanisms to an acidic shock are not identical among rhizobia species and may account for the acid tolerance of the strain, involving the induction of genes associated with different functional groups. Furthermore, our results have shown that a large number (approximately $16 \%$ ) of the responsive genes belong to the group of hypothetical or unknown genes. The present study can contribute for a better understanding of the molecular bases of response to acidic $\mathrm{pH}$, with the acidinduced genes representing promising targets for future investigations.

Acknowledgments This work was supported by Fundação para a Ciência e a Tecnologia (FCT), including the research projects FCOMP01-0124-FEDER-007091, FCOMP-01-0124-FEDER-028316 (PTDC/BIA-EVF/4158/2012), the strategic Project PEst-OE/AGR/ UI0115/2014 and InAlentejo Project ALENT-07-0262-FEDER-001871 that include FEDER funds through the Operational Programme for Competitiveness Factors-COMPETE and national funds. M. Laranjo and A. Alexandre acknowledge Post-Doc fellowships (SFRH/BPD/27008/2006 and SFRH/BPD/73243/2010) from FCT. The authors thank Ana Catarina Gomes from Biocant Park (Portugal) for her helpful guidance through 
microarray data analysis and Owen Woody from the University of Waterloo (Canada) for his help with the DNAplotter software.

\section{References}

Alexandre A, Oliveira S (2013) Response to temperature stress in rhizobia. Crit Rev Microbiol 39:219-228

Alexandre A, Laranjo M, Oliveira S (2014) Global transcriptional response to heat shock of the legume symbiont Mesorhizobium loti MAFF303099 comprises extensive gene downregulation. DNA Res 21:195-206

Audia JP, Webb CC, Foster JW (2001) Breaking through the acid barrier: an orchestrated response to proton stress by enteric bacteria. Int J Med Microbiol 291:97-106

Brígido C, Oliveira S (2013) Most acid-tolerant chickpea mesorhizobia show induction of major chaperone genes upon acid shock. Microb Ecol 65:145-153

Brígido C, Robledo M, Menendez E, Mateos PF, Oliveira S (2012) A ClpB chaperone knockout mutant of Mesorhizobium ciceri shows a delay in the root nodulation of chickpea plants. Mol Plant-Microbe Interact 25:1594-1604

Carver T, Thomson N, Bleasby A, Berriman M, Parkhill J (2009) DNAPlotter: circular and linear interactive genome visualization. Bioinformatics 25:119-120

Chen H, Gartner E, Rolfe BG (1993) Involvement of genes on a megaplasmid in the acid-tolerant phenotype of Rhizobium leguminosarum biovar trifolii. Appl Environ Microbiol 59:10581064

Chen WX, Wang ET, Kuykendall D (2005) Genus VI. Mesorhizobium. In: Garrity G, Brenner DJ, Krieg NR, Staley JT (eds) Bergeys manual of systematic bacteriology. vol 2 (The Proteobacteria). Springer, New York, pp 403-408

Correa OS, Barneix AJ (1997) Cellular mechanisms of $\mathrm{pH}$ tolerance in Rhizobium loti. World J Microbiol Biotechnol 13: $153-157$

Correa OS, Rivas EA, Barneix AJ (1999) Cellular envelopes and tolerance to acid $\mathrm{pH}$ in Mesorhizobium loti. Curr Microbiol 38:329-334

Cotter PD, Hill C (2003) Surviving the acid test: responses of Grampositive bacteria to low $\mathrm{pH}$. Microbiol Mol Biol Rev 67:429-453

Cunningham SD, Munns DN (1984) The correlation between extracellular polysaccharide production and acid tolerance in Rhizobium. Soil Sci Soc Am J 48:1273-1276

de Lucena DKC, Pühler A, Weidner S (2010) The role of sigma factor $\mathrm{RpoH} 1$ in the $\mathrm{pH}$ stress response of Sinorhizobium meliloti. BMC Microbiol 10:265

Dilworth MJ, Howieson JG, Reeve WG, Tiwari RP, Glenn AR (2001) Acid tolerance in legume root nodule bacteria and selecting for it. Aust J Exp Agric 41:435-446

Fenner BJ, Tiwari RP, Reeve WG, Dilworth MJ, Glenn AR (2004) Sinorhizobium medicae genes whose regulation involves the ActS and/or ActR signal transduction proteins. FEMS Microbiol Lett 236: 21-31

Foster JW (2001) Acid stress responses of Salmonella and E. coli: survival mechanisms, regulation, and implications for pathogenesis. J Microbiol 39:89-94

Foster JW (2004) Escherichia coli acid resistance: tales of an amateur acidophile. Nat Rev Microbiol 2:898-907

Glenn AR, Reeve WG, Tiwari RP, Dilworth MJ, Cook GM, Booth IR, Poole RK, Foster JW, Slonczewski JL, Padan E, Epstein W, Skulachev V, Matin A, Fillingame RH (1999) Acid tolerance in root nodule bacteria. In: Chadwick DJ, Cardew G (eds) Bacterial response to $\mathrm{pH}$. Novartis Foundation Symposium, vol 221. John Wiley \& Sons, Ltd, Chichester, pp 112-130
Goodson M, Rowbury RJ (1989) Habituation to normally lethal acidity by prior growth of Escherichia coli at a sub-lethal acid $\mathrm{pH}$ value. Lett Appl Microbiol 8:77-79

Götz S, García-Gómez JM, Terol J, Williams TD, Nagaraj SH, Nueda MJ, Robles M, Talón M, Dopazo J, Conesa A (2008) Highthroughput functional annotation and data mining with the Blast2GO suite. Nucleic Acids Res 36:3420-3435

Graham PH, Vance CP (2000) Nitrogen fixation in perspective: an overview of research and extension needs. Field Crop Res 65:93106

Graham PH, Draeger KJ, Ferrey ML, Conroy MJ, Hammer BE, Martinez E, Aarons SR, Quinto C (1994) Acid pH tolerance in strains of Rhizobium and Bradyrhizobium, and initial studies on the basis for acid tolerance of Rhizobium tropici UMR1899. Can J Microbiol 40: 198-207

Grkovic S, Brown MH, Skurray RA (2002) Regulation of bacterial drug export systems. Microbiol Mol Biol Rev 66:671-701

Gruber TM, Gross CA (2003) Multiple sigma subunits and the partitioning of bacterial transcription space. Annu Rev Microbiol $57: 441-466$

Hall HK, Karem KL, Foster JW (1995) Molecular responses of microbes to environmental $\mathrm{pH}$ stress. Adv Microb Physiol 37:229-272

Hellweg C, Pühler A, Weidner S (2009) The time course of the transcriptomic response of Sinorhizobium meliloti 1021 following a shift to acidic $\mathrm{pH}$. BMC Microbiol 9:37

Higgins CF (2001) ABC transporters: physiology, structure and mechanism - an overview. Res Microbiol 152:205-210

Kaneko T, Nakamura Y, Sato S, Asamizu E, Kato T, Sasamoto S, Watanabe A, Idesawa K, Ishikawa A, Kawashima K, Kimura T, Kishida Y, Kiyokawa C, Kohara M, Matsumoto M, Matsuno A, Mochizuki Y, Nakayama S, Nakazaki N, Shimpo S, Sugimoto M, Takeuchi C, Yamada M, Tabata S (2000) Complete genome structure of the nitrogen-fixing symbiotic bacterium Mesorhizobium loti. DNA Res 7:331-338

Kanjee U, Houry WA (2013) Mechanisms of acid resistance in Escherichia coli. Annu Rev Microbiol 67:65-81

Kaufusi PH, Forsberg LS, Tittabutr P, Borthakur D (2004) Regulation of exopolysaccharide synthesis in Rhizobium sp. strain TAL1145 involves an alternative sigma factor gene, $r p o H 2$. Microbiol-SGM 150:3473-3482

Laing E, Mersinias V, Smith C, Hubbard S (2006) Analysis of gene expression in operons of Streptomyces coelicolor. Genome Biol 7: R46

Laranjo M, Oliveira S (2011) Tolerance of Mesorhizobium type strains to different environmental stresses. Anton Leeuw Int J G 99:651-662

Laranjo M, Alexandre A, Oliveira S (2014) Legume growth-promoting rhizobia: an overview on the Mesorhizobium genus. Microbiol Res 169:2-17

Lee IS, Lin J, Hall HK, Bearson B, Foster JW (1995) The stationaryphase sigma factor $\sigma^{\mathrm{S}}(\mathrm{RpoS})$ is required for a sustained acid tolerance response in virulent Salmonella typhimurium. Mol Microbiol 17:155-167

Martín-Galiano AJ, Overweg K, Ferrandiz MJ, Reuter M, Wells JM, de la Campa AG (2005) Transcriptional analysis of the acid tolerance response in Streptococcus pneumoniae. Microbiol-SGM 151: 3935-3946

Masloboeva N, Reutimann L, Stiefel P, Follador R, Leimer N, Hennecke $\mathrm{H}$, Mesa S, Fischer H-M (2012) Reactive oxygen species-inducible ECF $\sigma$ factors of Bradyrhizobium japonicum. PLoS ONE 7:e43421

Österberg S, del Peso-Santos T, Shingler V (2011) Regulation of alternative sigma factor use. Annu Rev Microbiol 65:37-55

Pfaffl MW (2001) A new mathematical model for relative quantification in real-time RT-PCR. Nucleic Acids Res 29

Price MN, Huang KH, Alm EJ, Arkin AP (2005) A novel method for accurate operon predictions in all sequenced prokaryotes. Nucleic Acids Res 33:880-892 
Reverchon S, Van Gijsegem F, Effantin G, Zghidi-Abouzid O, Nasser W (2010) Systematic targeted mutagenesis of the MarR/SlyA family members of Dickeya dadantii 3937 reveals a role for MfbR in the modulation of virulence gene expression in response to acidic $\mathrm{pH}$. Mol Microbiol 78:1018-1037

Richardson AE, Simpson RJ, Djordjevic MA, Rolfe BG (1988) Expression of nodulation genes in Rhizobium leguminosarum biovar trifolii is affected by low $\mathrm{pH}$ and by $\mathrm{Ca}$ and $\mathrm{Al}$ ions. Appl Environ Microbiol 54:2541-2548

Ron EZ (2006) Bacterial stress response. In: Dworkin M, Falkow S, Rosenberg E, Schleifer K-H, Stackebrandt E (eds) Prokaryotes: a handbook on the biology of bacteria - ecophysiology and biochemistry. vol 2, 3rd edn. Springer, New York, pp 1012-1027

Saeed AI, Hagabati NK, Braisted JC, Liang W, Sharov V, Howe EA, Li J, Thiagarajan M, White JA, Quackenbush J (2006) TM4 microarray software suite. Methods Enzymol 411:134-193

Simon R, Lam A, Li M-C, Ngan M, Menenzes S, Zhao Y (2007) Analysis of gene expression data using BRB-ArrayTools. Cancer Informat 3:11-17

Szklarczyk D, Franceschini A, Kuhn M, Simonovic M, Roth A, Minguez P, Doerks T, Stark M, Muller J, Bork P, Jensen LJ, von Mering C (2011) The STRING database in 2011: functional interaction networks of proteins, globally integrated and scored. Nucleic Acids Res 39:D561-D568

Terpolilli JJ, Hood GA, Poole PS, Robert KP (2012) Chapter 5-what determines the efficiency of $\mathrm{N}_{2}$-fixing Rhizobium-legume symbioses? In: Poole RK (ed) Adv Microb Physiol. Vol 60. Academic Press, London, pp 325-389

Tiwari RP, Reeve WG, Dilworth MJ, Glenn AR (1996) Acid tolerance in Rhizobium meliloti strain WSM419 involves a two-component sensor-regulator system. Microbiol-SGM 142: 1693-1704

Tiwari RP, Reeve WG, Fenner BJ, Dilworth MJ, Glenn AR, Howieson JG (2004) Probing for pH-regulated genes in Sinorhizobium medicae using transcriptional analysis. J Mol Microbiol Biotechnol 7:133-139

Vincent JM (1970) A manual for the practical study of root-nodule bacteria, vol no. 15. Blackwell Scientific Publications, Oxford

Wren JD, Conway T (2006) Meta-analysis of published transcriptional and translational fold changes reveals a preference for low-fold inductions. OMICS: J Integr Biol 10:15-27

Zahran HH (1999) Rhizobium-legume symbiosis and nitrogen fixation under severe conditions and in an arid climate. Microbiol Mol Biol Rev 63:968-989 